\title{
CUESTIONES FUNDAMENTALES DE DIRECCIÓN DE OPERACIONES EN LA DIRECCIÓN Y GESTIÓN HOTELERA
}

\author{
Cristina Ceballos Hernández ${ }^{1}$ \\ Departamento de Economía Financiera y Dirección de Operaciones \\ Universidad de Sevilla \\ cceballos@us.es \\ Antonio Ruiz Jiménez ${ }^{2}$ \\ Departamento de Economía Financiera y Dirección de Operaciones \\ Universidad de Sevilla \\ anruiz@us.es
}

\begin{abstract}
Resumen
La investigación realizada tiene como propósito general enfocar la enseñanza de Dirección de Operaciones en la Universidad a las necesidades reales del sector. En este trabajo nos centramos en el estudio realizado en el ámbito empresarial, tratando de responder a la pregunta ¿cuáles son las necesidades del sector hotelero en lo que respecta a la formación en Dirección de Operaciones? Tras aplicar el método Delphi, se presentan los aspectos percibidos como más importantes por los expertos. Entre otras, destacan la medición de la satisfacción del cliente y la adecuada planificación y programación de los recursos humanos como tareas o cuestiones fundamentales en la dirección y gestión de estas empresas. Los resultados que se presentan podrían servir para orientar el contenido a impartir sobre Dirección de Operaciones en el ámbito universitario (grados y postgrados) si se pretende atender tales necesidades.
\end{abstract}

\section{Palabras clave}

dirección de operaciones, sector hotelero, necesidades, universidad, estudio Delphi

\begin{abstract}
The objective of our research is to focus the teaching of Operations Management at the University to the real needs of the sector. In this paper we focus in hospitality industry. We try to answer the following question: what are the needs of the hospitality industry in relation to tourism training in Operations Management? Delphi method is used. Some of the main issues perceived as most important by the experts are, for example: measurement of customer satisfaction and planning and scheduling of human resources. In our opinion, the results presented could be used to guide the tourism training at the University.
\end{abstract}

\section{Keywords}

operations management, hospitality industry, real needs, university, Delphi method

\section{INTRODUCCIÓN}

Estamos plenamente convencidos de la conveniencia que tiene que la investigación universitaria esté en íntima conexión con el sector real de nuestra economía. Asimismo, consideramos necesario que las enseñanzas que se impartan traten de reflejar lo que el sector demanda, de forma que la formación universitaria tenga suficiente valor y unas mínimas garantías que no supongan necesariamente una reeducación del personal que se incorpora, con los costes adicionales que puede suponer para la empresa. En ocasiones, es el propio egresado el que se hace cargo de dicha formación extra, con el fin de diseñar un perfil curricular más ajustado a las necesidades del entorno empresarial.

Es en esta línea donde se encuadra la investigación que los autores vienen desarrollando. Con ella tratamos de enfocar la enseñanza que se imparte en las universidades, en lo que al sector turístico se refiere, a las necesidades que realmente presenta dicho sector. Nos centramos en el área de Operaciones de la empresa, por ser nuestra área de trabajo e investigación y por el convencimiento de que la buena gestión de las operaciones en las empresas de servicios, y en las turísticas en particular, constituye una fuente de ventaja competitiva. El estudio empírico desarrollado ha tenido dos partes: una con profesores, dedicada a conocer el estado de la enseñanza de Dirección de Operaciones en los estudios universitarios de Turismo, y otra encaminada a conocer qué necesidades presenta al respecto el sector hotelero. En el artículo se recoge esta última parte del estudio y algunos de los resultados obtenidos. En concreto respondemos a la pregunta: ¿cuál es la importancia que para

\footnotetext{
${ }^{1}$ Doctora por la Universidad de Sevilla

2 Doctor en Administración de Empresas
} 
la adecuada dirección y gestión hotelera tienen distintos aspectos encuadrados dentro del ámbito de la Dirección de Operaciones? ¿Cuáles son las cuestiones fundamentales?

\section{MARCO TEÓRICO}

La formación en el sector turístico sigue siendo objeto de numerosos debates a nivel nacional e internacional por diversos motivos. Entre ellos podríamos señalar el amplio consenso que existe sobre el importante papel que la formación del capital humano juega en la consecución de altas eficiencias y de valor añadido en las empresas de servicios, en general, y del sector turístico, en particular, intensivos, como sabemos, en mano de obra (Onyango et al., 2009). Por otro lado, cada vez somos más conscientes de que la formación de los empleados del sector afecta directamente a la calidad de la industria del turismo de un país (ver, por ejemplo, Gestión de Hoteles, 2008, p.40; Lillo y otros, 2007, p. 63; Talón et al., 2007, p.12; Küster y Antón, 2004, p. 51; entre otros). Por último, las características del entorno en que se desarrollan las actividades turísticas (entre ellas, globalización de los mercados, la creciente competitividad, la segmentación de la demanda, las nuevas tecnologías, los requisitos de sustentabilidad ambiental y social, etc.) exigen una adaptación continua de los contenidos en la formación del capital humano (Zehrer y Mössenlechner, 2008; Huete, 2007; Zehrer et al., 2006).

A pesar de esta reconocida importancia, continúa existiendo un debate abierto sobre muchos aspectos relacionados con las necesidades de formación de los recursos humanos en las organizaciones del sector turístico. Uno de los aspectos que más interés suscita dentro de la formación en Turismo es el contenido de los planes de estudio, el cual ha dado lugar a no pocas investigaciones a nivel internacional y nacional (ver Ceballos y Ruiz, 2009, p. 55-67). Y aunque también se han realizado algunos estudios sobre el contenido de materias concretas, no existen, investigaciones previas sobre la enseñanza de Dirección de Operaciones en Turismo.

Por otro lado, cuando nos dirigimos al ámbito empresarial, comprobamos que los estudios realizados entre las empresas del sector terciario y, en particular, en aquéllas que pertenecen al sector turístico, sobre los diversos aspectos de la Dirección de Operaciones, técnicas, problemas, etc. son mucho más escasos que los realizados en las empresas industriales, además de poco recientes. Esto es así aun cuando parecen claros los beneficios que para las empresas de servicios puede tener una adecuada gestión de sus operaciones para conseguir aumentos en la eficiencia y competitividad. En este sentido podemos destacar los trabajos de De Burgos et al. (2003), Wright y Mechling (2002), Álvarez et al. (2001), Witt y Clark (1990) y Witt y Witt (1989). A pesar de los estudios previos sobre la praxis de la Dirección de Operaciones en el sector hotelero, hemos comprobado que ninguno de ellos está encaminado a determinar las necesidades de estas empresas y su comparación con la enseñanza que se imparte en las universidades, lo que sí constituye el objetivo de nuestra investigación. A continuación, presentamos la metodología que hemos empleado en el estudio empírico desarrollado en el sector hotelero: el Método Delphi.

\section{METODOLOGÍA}

La técnica Delphi es un método de consulta ampliamente aceptado que permite, de forma rápida y eficaz, recoger opiniones de expertos y conseguir el consenso de un grupo de personas que tienen conocimientos sobre factores de carácter desconocido, temas subjetivos o problemas complejos (Garrod y Fyall, 2005; Green et al., 1990; Linstone y Turoff, 1975). Como señalan Kerstetter y Cho (2004, p. 964), los datos recogidos a través de un estudio Delphi son de buena calidad, ya que proceden de expertos perfectamente capaces de apreciar la complejidad del problema y preparados para tratarlos y analizarlos.

La técnica Delphi ha sido ampliamente utilizada para recoger opiniones sobre el contenido de la formación que se considera adecuada sobre una materia o estudios en concreto. Muchos de estos trabajos previos han tenido, como objetivo último, proponer un contenido que sirva de referencia en el diseño de planes de estudios o programas de formación (Kim et al., 2013; May et al., 2013; Kunina-Habenich et al., 2012). En el sector turístico también se ha empleado dicha técnica para la determinación de las necesidades formativas sobre diversos aspectos (finanzas, sistemas de información, competencias generales, etc.), en diferentes niveles formativos (enseñanza secundaria, grados, cursos de doctorado, etc.) y a distintos niveles profesionales (personal base, directivos, etc.) (ver, por ejemplo, Masberg et al.2003; Breiter y Hoart, 2000; Chernish y Spivack, 1998; FayosSola, 1997).

A continuación, explicamos algunos aspectos clave de la aplicación de la metodología Delphi en nuestra investigación:

\subsection{Panel de expertos y área geográfica del estudio}

Los miembros que conformen el panel deben ser seleccionados por tener cierto interés en el tema o bien por poseer conocimientos relevantes sobre el mismo (Spenceley, 2008). Los criterios que se definan para seleccionarlos pueden hacer referencia al nivel de conocimiento que posea, a su experiencia o su localización geográfica entre otros, aunque poseer una experiencia mínima acreditada debe ser, según algunos autores, el principal requisito para seleccionar los expertos de un panel (Gallego Pereira et al. 2008; Zolingen y Klaassen, 2003). 
Tabla 1: Cadenas hoteleras que han participado en el estudio Delphi

\begin{tabular}{|c|c|}
\hline Cadena hotelera & $\begin{array}{c}\text { Puesto en el ranking de cadenas } \\
\text { españolas }\end{array}$ \\
\hline Sol Meliá & 1 \\
\hline $\mathrm{NH}$ & 2 \\
\hline Barceló & 3 \\
\hline Occidental Hotels \& Resorts & 10 \\
\hline AC Hotels & 13 \\
\hline Hoteles Catalonia & 15 \\
\hline Vincci Hoteles & 23 \\
\hline Hoteles Silken & 24 \\
\hline Hoteles Globales & 29 \\
\hline Med Playa & 36 \\
\hline ABBA Hoteles & 37 \\
\hline Hoteles Center & 58 \\
\hline Room Mate Hoteles & 72 \\
\hline Hoteles Monte & 73 \\
\hline Hoteles, Casas y Palacios de España & - \\
\hline MS Hoteles & - \\
\hline Stabotel & - \\
\hline
\end{tabular}

En nuestro caso, como paso previo a la selección de los expertos que formarían el panel, consideramos que las principales cadenas hoteleras a nivel nacional debían estar presente en el mismo, haciendo corresponder dicho ámbito geográfico al establecido en la otra parte del estudio empírico, es decir, el llevado a cabo con profesores. Decidimos tomar como punto de partida el "Ranking global de establecimientos y habitaciones" publicado por Hosteltur y compuesto por un total de 84 cadenas $^{3}$. A partir de la bibliografía consultada, se establecieron unos criterios previos (en cuanto al cargo ocupado, años de experiencia profesional, tamaño del establecimiento, etc.). A continuación, se invitó a participar a expertos de estas 84 cadenas, aplicándose además un muestreo bola de nieve mediante el cual también participaron finalmente en el estudio otros expertos, de contrastada experiencia, pero de cadenas hoteleras no incluidas en el ranking anterior.

Nuestro panel inicial estuvo finalmente conformado por 33 expertos, valor algo superior incluso al rango recomendado por muchos autores y mayor que el empleado en estudios previos con similares fines (ver Ceballos y Ruiz, 2009, p.562). Estos expertos pertenecen a 17 cadenas hoteleras diferentes (ver Tabla 1). Por último destacar que los panelistas dirigen establecimientos distribuidos a lo largo del territorio nacional, entre ellos en: Cádiz, Málaga, Barcelona, Madrid, Palma de Mallorca, Salamanca, Sevilla, etc.

\subsection{Diseño del cuestionario}

En el cuestionario utilizado se identifican dos partes: una primera donde se incluyen datos personales del experto y del establecimiento y algunas preguntas sobre su experiencia profesional y académica, y una segunda relacionada con la Dirección de Operaciones en la dirección y gestión hotelera.

Esta segunda parte incluía varias secciones, una de ellas con un listado de aspectos de Dirección de Operaciones sobre los cuales los expertos debían indicar la importancia que tienen en la correcta dirección y gestión de establecimientos hoteleros. Lógicamente dicha relación de aspectos se correspondía con el listado de temas empleado en el ámbito universitario, de forma que la comparación de los ámbitos académico y empresarial resultara válida. Para construir este listado recurrimos a estudios previos sobre el tema, y se consultaron numerosos manuales de especial relevancia en esta disciplina, así como otros que abordan el estudio de la Dirección de Operaciones en servicios y manuales o estudios sobre la Dirección de Operaciones en el sector turístico (ver Ceballos y Ruiz, 2009, p. 223-233 y 277-278). El listado ha incluido un total de 12 aspectos estratégicos y 15 táctico/operativos. Para su valoración se empleó una escala tipo Likert de cinco puntos, con el siguiente significado: (1) Sin importancia o importancia muy baja y (5) Importancia muy alta. Validado el cuestionario el mismo se envió por correo postal en las distintas rondas.

\subsection{Análisis de datos y retroalimentación}

Para cada una de las rondas se prepararon los cuestionarios en papel. Para cada ítem incluimos la respuesta del experto en comparación con la respuesta grupal. Como valor agregado de la misma indicamos la media, la mediana y la desviación típica, tal y como se hace en estudios previos (Briedenhann, 2007) y atendiendo a las

\footnotetext{
${ }^{3}$ Hosteltur (2008): "Ranking Hoteltur de cadenas hoteleras españolas 2008". Hosteltur. Septiembre 2008, p. 29.
} 
recomendaciones de un estadístico. Fueron resaltados además aquellas cuestiones en las que la opinión del experto se alejaba más de la respuesta grupal. Estas fueron todas aquellas que se encontraban fuera del rango intercuartílico y más alejadas de la media, lo que supuso elaborar un cuestionario personalizado para cada experto.

\subsection{Número de rondas}

Tras realizar la primera ronda no se alcanzó el nivel de consenso establecido (rango intercuartilico menor e igual que 1,5 en al menos el $85 \%$ de los ítems). En algunos casos la desviación típica era elevada y el rango intercuartílico bastante amplio. Esto indicaba la necesidad de realizar una segunda ronda. Después de la misma se observó una reducción en las desviaciones típicas de algunas variables, estrechándose también el rango intercuartílico en varias de ellas. Pudimos comprobar como más del $85 \%$ de los ítems presentaban ahora un rango intercuartílico menor o igual que 1,5. Asimismo, se apreciaba una cierta estabilidad en las respuestas, ya que las variaciones observadas en la desviación típica y el rango intercuartílico no habían sido demasiado significativas, aunque si suficientes como para converger y alcanzar el nivel de consenso establecido. De acuerdo con algunos autores como Briedenhann (2007) un movimiento leve hacia el acuerdo general entre rondas no hace necesario una consulta adicional, por lo que la tercera ronda consultiva no se consideró necesaria.

De los 33 expertos iniciales, un total de 27 participaron en la segunda ronda y finalizaron el estudio, lo que supone una elevada tasa de participación $(81,8 \%)$, en comparación con la alcanzada en otras investigaciones Delphi desarrolladas en el sector turístico (p.e. Briedenhann, 2007; O'Connor y Frew, 2004) o con estudios sobre necesidades formativas (p.e. Rohan et al., 2009; Sulzer-Azaroff et al., 2008).

En el siguiente apartado se presentan algunos de los resultados obtenidos en nuestro estudio.

\section{RESULTADOS}

Podemos comenzar este apartado comentando algunos aspectos relevantes del panel de expertos utilizado en la investigación. Como se observa en la Tabla 2, el 85,2\% de ellos tiene una experiencia superior a 10 años y el $51,9 \%$ de más de 15 . Además, aunque no es una variable determinante en base a estudios previos, podemos decir que en nuestro caso, cuando los expertos dirigen establecimientos (que son el $74,1 \%$ de ellos), estos son de 4 ó 5 estrellas en el $90 \%$ de los casos. Hemos de resaltar este hecho, si tenemos en cuenta que la categoría del establecimiento está relacionada con una gestión "más científica" de las operaciones y con una mayor aplicación de las nuevas tecnologías (Ceballos Hernández et al., 2008), aspecto importante cuando muchas de las técnicas de Dirección de Operaciones necesitan apoyarse en las mismas. Añadido a esto, los establecimientos de mayor tamaño suelen ser, en términos generales, de mayor categoría ${ }^{4}$. Este aspecto es importante en nuestro caso porque estudios previos han señalado una relación positiva entre el tamaño y la aplicación de las técnicas de Dirección de Operaciones (De Burgos et al., 2003; Álvarez et al., 2001; Witt y Clark, 1990; Witt y Witt, 1989).

Tabla 2: Perfil del panel

\begin{tabular}{|c|c|c|}
\hline Ítem & Descripción variable & $\mathbf{N}(\%)$ \\
\hline \multirow{7}{*}{$\begin{array}{l}\text { Datos } \\
\text { personales }\end{array}$} & Sexo & \\
\hline & Hombre & $20(74,1 \%)$ \\
\hline & Mujer & $7(25,9 \%)$ \\
\hline & Cargo & \\
\hline & Director de hotel de 4 ó 5 estrellas & $18(66,7 \%)$ \\
\hline & Director de hotel de 3 estrellas & $2(7,4 \%)$ \\
\hline & Otros puestos directivos & $7(25,9 \%)$ \\
\hline \multirow{5}{*}{ Ítem P.1 } & Antigüedad en puestos directivos & \\
\hline & Menos de 5 & $1(3,7 \%)$ \\
\hline & De 5 a 10 & $3(11,1 \%)$ \\
\hline & De 11 a 15 & $9(33,3 \%)$ \\
\hline & Más de 15 & $14(51,9 \%)$ \\
\hline \multirow{5}{*}{ Ítem P.2 } & Antigüedad en puesto actual & \\
\hline & Menos de 5 & $10(37,0 \%)$ \\
\hline & De 5 a 10 & $6(22,2 \%)$ \\
\hline & De 11 a 15 & $2(7,4 \%)$ \\
\hline & Más de 15 & $9(33,3 \%)$ \\
\hline \multirow{5}{*}{ Ítem P.3 } & Experiencia docente & $\mathrm{N}(\%)$ \\
\hline & $\begin{array}{r}\text { Colabora o ha colaborado en labores } \\
\text { docentes a nivel universitario }\end{array}$ & $11(40,7 \%)$ \\
\hline & $\begin{array}{r}\text { Colabora o ha colaborado en labores } \\
\text { docentes a nivel NO universitario }\end{array}$ & $8(29,6 \%)$ \\
\hline & $\begin{array}{r}\text { Colabora o ha colaborado en labores } \\
\text { docentes a nivel universitario y no } \\
\text { universitario }\end{array}$ & $2(7,4 \%)$ \\
\hline & $\begin{array}{r}\text { No colabora ni ha colaborado en labores } \\
\text { docentes }\end{array}$ & $6(22,2 \%)$ \\
\hline
\end{tabular}

\footnotetext{
${ }^{4} \mathrm{~A}$ partir del número de establecimientos y de plazas según las categorías recogidos anualmente en la Encuesta de Ocupación Hotelera (INE), se observa un mayor tamaño, por término medio, en los establecimientos de mayor categoría.
} 
El ítem P.3 de la Tabla 2 hace referencia a la experiencia docente de los expertos del panel. Consideramos que este aspecto es relevante en un estudio en el que se pretenden determinar las necesidades formativas en el sector y proponer, a partir de estas, un contenido para la enseñanza de Dirección de Operaciones en la Universidad. A este respecto hemos de señalar que la mayor parte de los expertos seleccionados tienen algún tipo de experiencia docente a nivel universitario, concretamente el $40,7 \%$, y casi un $30 \%$ a nivel no universitario, en la enorme mayoría de los casos en másteres de carácter profesional. A esto hay que sumar el 7,4\% que indican haber colaborado en actividades docentes a ambos niveles. Esto nos lleva a concluir que se trata de un panel conformado por expertos con una amplia experiencia profesional y una experiencia académica en casi el $80 \%$ de los casos.

A continuación, nos centramos en la valoración que los expertos realizan sobre la importancia de los aspectos de Dirección de Operaciones en la adecuada dirección y gestión hotelera. Se realiza un análisis conjunto tanto de aspectos estratégicos como táctico/operativos, un total de 27 (Tabla 3). Como puede observarse, las valoraciones oscilan entre 4,78 y 3,42 (recordemos en una escala de 1 a 5), lo que pone de nuevo de relieve la importancia considerable de estos aspectos en la adecuada dirección y gestión hotelera en opinión de los profesionales consultados. Atendiendo a dichos valores máximos y mínimos, los aspectos se han clasificado en tres bloques: "Nivel 1" (puntuación comprendida entre 4,78 y 4,33), "Nivel 2" (puntuación entre 4,32 y 3,88) y "Nivel 3" (puntuación entre 3,87 y 3,42). Aquellos aspectos en los que no se ha alcanzado el nivel de acuerdo establecido se han marcado con un asterisco $\left({ }^{*}\right)$.

Podemos comprobar que entre los aspectos que reciben una mayor importancia se encuentran bastantes de los que hemos clasificado como aspectos tácticos/operativos (ver columna E/TO). El cliente parece ser uno de los principales objetivos de estas empresas, ya que medir su satisfacción se convierte en el aspecto más importante $(4,78)$. La satisfacción del cliente es claramente una fuente de ventajas competitivas en el sector hotelero, de ahí la importancia que recibe.

Muy cerca de este aspecto, y ocupando el segundo lugar en importancia en la tabla, encontramos la necesidad de planificar y programar los recursos humanos de la organización $(4,74)$. Este aspecto está relacionado con la naturaleza propia de los servicios, intensivos como sabemos en mano de obra. Además, es innegable la importancia que alcanza el diseño de turnos de trabajo en los establecimientos hoteleros, de forma que se asegure, con los mismos, el funcionamiento permanente de un negocio de tales características, el cual se encuentra "despierto" 24 horas al día y está orientado totalmente a atender las necesidades del cliente.

Como recogen muchos autores, entre ellos Jones et al. (2007), el nivel de satisfacción viene determinado por la calidad percibida por el cliente, aunque esta relación, ampliamente investigada, ha sido objeto de algunas cítricas y limitaciones en lo que se refiere a las dimensiones que la conforman y a las técnicas para su cuantificación (Fernández García et al., 2001). Observando las puntuaciones recibidas en la Tabla 3, podemos decir que los aspectos relacionados con la calidad, en todo lo relativo al diseño, gestión y control de la misma, reciben una relativa importancia (con una puntuación media de 4,37 para los aspectos táctico/operativos y 4,30 para los estratégicos, $R=9$ y $R=13$ respectivamente). De nuevo se observa una mayor preocupación por el medio y corto plazo. Los factores determinantes de la calidad, según estudios previos, parecen estar relacionados con factores tangibles e intangibles que generan valor para el cliente, la puntualidad y las instalaciones del hotel (Jones et al., 2007). Esto atribuye una especial importancia al diseño del servicio y de su prestación. De esta forma comprobamos como definir una adecuada estrategia de operaciones $(4,63)$ y, dentro de esta, seleccionar y diseñar correctamente el servicio $(4,67)$, son otros de los aspectos más importantes a la vista del ranking $(R=4$ y $\mathrm{R}=3$ respectivamente). Estos, junto con el diseño del proceso de prestación del servicio $(4,56$, $R=7)$, se convierten en los aspectos a largo plazo más relevantes para el sector. 
Tabla 3. Ranking conjunto de aspectos Dirección de Operaciones en el sector hotelero según la importancia media (*) Items en los que no se ha alcanzado el nivel de acuerdo establecido

\begin{tabular}{|c|c|c|c|c|c|c|c|}
\hline $\mathbf{R}$ & E-T/O & ASPECTOS DIRECCIÓN OPERACIONES & $\stackrel{\frac{\pi}{\mathrm{d}}}{\Sigma}$ & 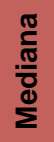 & 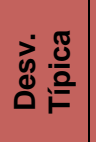 & $\mathbf{N}$ & 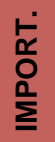 \\
\hline 1 & $\mathrm{~T} / \mathrm{O}$ & Medir la satisfacción del cliente & 4,78 & 5 & 0,42 & 27 & \multirow{12}{*}{ N1 } \\
\hline 2 & $\mathrm{~T} / \mathrm{O}$ & $\begin{array}{l}\text { Planificar y programar adecuadamente los recursos humanos } \\
\text { de la organización }\end{array}$ & 4,74 & 5 & 0,81 & 27 & \\
\hline 3 & $\mathrm{E}$ & $\begin{array}{l}\text { Realizar una correcta selección y diseño de los servicios a } \\
\text { ofrecer }\end{array}$ & 4,67 & 5 & 0,48 & 27 & \\
\hline \multirow[t]{2}{*}{4} & $\mathrm{E}$ & $\begin{array}{l}\text { Definir una adecuada Estrategia de Operaciones así como sus } \\
\text { objetivos a largo plazo }\end{array}$ & 4,63 & 5 & 0,56 & 27 & \\
\hline & $\mathrm{T} / \mathrm{O}$ & Medir el rendimiento y la productividad & 4,63 & 5 & 0,49 & 27 & \\
\hline 6 & $\mathrm{~T} / \mathrm{O}$ & $\begin{array}{l}\text { Gestionar de forma correcta la demanda y la capacidad a } \\
\text { medio y corto plazo. Aplicar Yield Management }\end{array}$ & 4,59 & 5 & 0,89 & 27 & \\
\hline \multirow[t]{2}{*}{7} & $\mathrm{~T} / \mathrm{O}$ & $\begin{array}{l}\text { Planificar, programar y controlar adecuadamente las } \\
\text { operaciones a medio y corto plazo }\end{array}$ & 4,56 & 5 & 0,51 & 27 & \\
\hline & $\mathrm{E}$ & $\begin{array}{l}\text { Seleccionar y diseñar el sistema de prestación del servicio de } \\
\text { manera adecuada }\end{array}$ & 4,56 & 5 & 0,58 & 27 & \\
\hline \multirow[t]{2}{*}{9} & $\mathrm{~T} / \mathrm{O}$ & $\begin{array}{l}\text { Adoptar las medidas táctico/operativas adecuadas } \\
\text { relacionadas con la gestión y control de la calidad }\end{array}$ & 4,37 & 5 & 0,79 & 27 & \\
\hline & $\mathrm{T} / \mathrm{O}$ & Controlar los procesos & 4,37 & 5 & 0,74 & 27 & \\
\hline \multirow[t]{2}{*}{11} & $\mathrm{E}$ & Diseñar correctamente los sistemas de trabajo & 4,33 & 5 & 0,88 & 27 & \\
\hline & $\mathrm{T} / \mathrm{O}$ & $\begin{array}{l}\text { Realizar una adecuada gestión medioambiental y adoptar } \\
\text { sistemas integrados para la gestión de ésta junto con la } \\
\text { calidad y riesgos laborales. }\end{array}$ & 4,33 & 4 & 0,68 & 27 & \\
\hline \multirow[t]{3}{*}{13} & $\mathrm{E}$ & Distribuir correctamente el espacio físico de las instalaciones & 4,30 & 4 & 0,87 & 27 & \multirow{10}{*}{ N2 } \\
\hline & $\mathrm{E}$ & $\begin{array}{l}\text { Otorgar la debida importancia estratégica al diseño y gestión } \\
\text { de la calidad }\end{array}$ & 4,30 & 4 & 0,78 & 27 & \\
\hline & $\mathrm{E}$ & Utilizar de forma adecuada las nuevas tecnologías & 4,30 & 4 & 0,78 & 27 & \\
\hline 16 & $\mathrm{E}$ & $\begin{array}{l}\text { Dirigir correctamente las cuestiones estratégicas referidas a la } \\
\text { cadena de suministro y la logística }\end{array}$ & 4,19 & 4 & 0,88 & 27 & \\
\hline 17 & $\mathrm{~T} / \mathrm{O}$ & $\begin{array}{l}\text { Realizar una correcta planificación agregada y programación } \\
\text { maestra }\end{array}$ & 4,13 & 4 & 0,61 & 24 & \\
\hline 18 & $\mathrm{E}$ & Adoptar adecuadamente las decisiones de localización & 4,12 & 4 & 0,71 & 26 & \\
\hline 19 & $\mathrm{~T} / \mathrm{O}$ & $\begin{array}{l}\text { Adoptar las decisiones táctico/operativas correctas sobre la } \\
\text { cadena de suministro y logística }\end{array}$ & 4,11 & 4 & 0,64 & 27 & \\
\hline 20 & $\mathrm{~T} / \mathrm{O}$ & Gestionar convenientemente los inventarios & $4,04^{*}$ & 4 & 0,98 & 27 & \\
\hline \multirow[t]{2}{*}{21} & $\mathrm{E}$ & $\begin{array}{l}\text { Conocer las características que distinguen a las } \\
\text { organizaciones de servicios de aquellas otras que fabrican } \\
\text { bienes, así como la importancia de las operaciones en la } \\
\text { dirección y gestión hotelera. }\end{array}$ & 3,96 & 4 & 0,76 & 27 & \\
\hline & $\mathrm{T} / \mathrm{O}$ & Gestionar de forma oportuna los proyectos & 3,96 & 4 & 0,71 & 27 & \\
\hline 23 & $\mathrm{~T} / \mathrm{O}$ & Aplicar sistemas MRP/ERP & 3,85 & 4 & 0,75 & 20 & \multirow{5}{*}{ N3 } \\
\hline 24 & $\mathrm{~T} / \mathrm{O}$ & $\begin{array}{l}\text { Utilizar adecuadamente las enseñanzas de la filosofía Justo a } \\
\text { Tiempo/Lean Production }\end{array}$ & $3,75^{\star}$ & 4 & 1,03 & 24 & \\
\hline \multirow[t]{2}{*}{25} & $E$ & $\begin{array}{l}\text { Planificar correctamente la capacidad a largo plazo y diseñar } \\
\text { un sistema para la gestión de colas }\end{array}$ & 3,63 & 4 & 0,77 & 24 & \\
\hline & $E$ & $\begin{array}{l}\text { Tener la debida consideración hacia los fenómenos de } \\
\text { internacionalización y globalización en las empresas turísticas }\end{array}$ & 3,63 & 4 & 0,93 & 27 & \\
\hline 27 & $\mathrm{~T} / \mathrm{O}$ & $\begin{array}{l}\text { Utilizar adecuadamente las enseñanzas de la Teoría de las } \\
\text { Limitaciones (TOC/OPT) }\end{array}$ & 3,42 & 3 & 0,84 & 19 & \\
\hline
\end{tabular}


También en cuarto lugar, con una puntuación bastante alta de 4,63, encontramos la medición del rendimiento y la productividad. La preocupación de las empresas de servicios por incrementar los niveles de productividad, ha dado lugar a no pocas investigaciones que han derivado en propuestas de instrumentos de medición (como el $\left.D E A^{5}\right)$ así como distintos indicadores, como por ejemplo el Revpar. Entre los realizados en el sector hotelero, podemos señalar algunos como, por ejemplo, Zhou et al. (2008); Sigala et al. (2005) y Hu y Cai (2004).

Otro de los aspectos claves en la dirección y gestión hotelera radica en la gestión de la demanda y la capacidad a nivel táctico y operativo $(4,59, \mathrm{R}=6)$, que en concreto incluiría todo lo relacionado con el Yield Management. Llama la atención el desigual tratamiento cuando hablamos del largo plazo, a pesar del carácter no inventariable y perecedero de los servicios hoteleros que, suponemos, lo hace meritorio de una atención especial en la gestión realizada a todos los niveles. Como se aprecia en la Tabla 3, la planificación de la capacidad a largo plazo y el diseño de sistemas de colas, aparecen como uno de los aspectos menos relevantes para el sector, ocupando uno de los lugares que cierran la tabla y situándose entre los de Nivel 3 (puntuación 3,63).

Como sabemos, algunas ideas erróneas sobre el carácter inofensivo del turismo en el medioambiente, han dado lugar a una lenta integración de las consideraciones ambientales y sociales en la planificación y desarrollo del turismo, así como en la gestión de las empresas. De esta forma no es hasta finales de los años 80 cuando se comienzan a abordar estas cuestiones desde la industria (Kasim, 2006). Actualmente, podemos decir que existe una preocupación creciente acerca de los efectos perjudiciales que las actividades desarrolladas por las empresas hoteleras tienen en el medio ambiente. Cada vez en mayor medida, estas organizaciones están siendo conscientes del grado en que interactúan con el medio, en lo que se refiere al consumo de recursos y a la generación de residuos, y de los efectos que de ambas se derivan. Que duda cabe, además, de que las consideraciones medioambientales en la gestión hotelera pueden llevar a conseguir ventajas competitivas sostenibles para la empresa. Las investigaciones desde el ámbito académico realizadas sobre el tema son también cada vez más numerosas, poniendo de nuevo esto de relieve la importancia de combinar, de forma adecuada, un turismo sostenible y los servicios de alojamiento (ver, por ejemplo, Chan et al., 2008). Desde el sector hotelero, podemos decir que cada vez son más las iniciativas tomadas por las grandes cadenas, incluyendo en su funcionamiento las consideraciones ambientales, aunque lo cierto es que no se observa tanta evidencia en las empresas de menor tamaño (Kasim, 2007).

Según los resultados obtenidos, estos expertos atribuyen una importancia media de 4,33 (R=10) a la gestión medioambiental y la adopción de sistemas integrales de calidad, medioambiente y riesgos laborales. Esta puntuación, puede resultar elevada atendiendo a la escala utilizada, aunque también es cierto que en nuestro estudio se identifican otros muchos aspectos con una importancia mayor.

También observamos en la Tabla 3 la escasa importancia, en relación con otros, que algunos temas estratégicos parecen tener en la dirección y gestión de establecimientos hoteleros. Nos referimos a aspectos tradicionalmente asociados a la gestión del área de Operaciones a este nivel, como las decisiones de distribución en planta $(4,30$, $R=13$ ) y de localización (4,12, $R=18)$, ambos en el Nivel 2.

Algo parecido sucede con la gestión de inventarios, que debido al carácter intangible de los servicios recibe una escasa atención y se sitúa en la segunda mitad de la tabla $(R=20)$. Las cuestiones tanto estratégicas como táctico/operativas relacionadas con la cadena de suministros, tampoco parecen ocupar uno de los puestos más relevantes en opinión de los expertos. Con una puntuación media de 4,19 y 4,11, se sitúan en los puestos 16 y 19 , respectivamente.

Por último, consideramos conveniente hacer mención a los aspectos relacionados con los fenómenos de internacionalización y globalización de la empresa, puesto que los mismos acaparan poca atención en el panel y se sitúan en los últimos puestos del ranking.

Finalizamos destacando que en los temas estratégicos y táctico/operativos en general se aprecia una desviación típica bastante pequeña, sobre todo si comparamos estos valores con los alcanzados en otros bloques o variables concreta del estudio. Esto indica una escasa desviación de las respuestas con respecto al valor medio. Se aprecia consenso en la importancia asignada a cada uno de estos aspectos excepto en dos de ellos, en los que el rango intercuartílico supera el valor de 1,5 establecido como límite (estos valores aparecen destacados con un asterisco). También la desviación típica es alta en ambos casos. Este similar comportamiento parece lógico al tratarse de aspectos relacionados, ya que la adecuada gestión del inventario es uno de los aspectos clave en la filosofía Justo a Tiempo.

\section{CONCLUSIONES}

Con este trabajo hemos tratado de determinar cuáles son los distintos aspectos o temas relacionados con la Dirección de Operaciones que el sector hotelero percibe como más importantes en la adecuada dirección y gestión hotelera. Para ello se ha llevado a cabo un estudio Delphi de dos rondas en el que han participado 27 profesionales del sector hotelero. En cuanto al panel de expertos se observa que el $74,1 \%$ son directores de hotel, en su mayoría en establecimientos de 4 o más estrellas, mientras que el resto ocupan otros puestos directivos en el sector. Los expertos tienen, además, una amplia experiencia profesional (el 85,2\% llevan más de 10 años ocupando puestos directivos, siendo el 51,9\% los que llevan más de 15) y una experiencia académica en casi el $80 \%$ de los casos, principalmente en el ámbito universitario.

En 25 de los 27 aspectos valorados en el estudio (92,6\%) se ha alcanzado el nivel de consenso establecido. Las puntuaciones que reciben estas cuestiones de Dirección de Operaciones se pueden considerar en general altas,

\footnotetext{
${ }^{5}$ Data Envelopment Analysis (DEA) o Análisis Envolvente de Datos.
} 
lo que pone de manifiesto la importancia que estos temas tienen en la dirección y gestión hotelera y, por tanto, la necesaria inclusión de los mismos en la formación universitaria si se pretende responder a las necesidades del sector. Los expertos consultados parecen otorgar una mayor importancia a muchos de los aspectos táctico/operativos relativos a la Dirección de Operaciones, pues de los diez que reciben una mayor importancia, siete corresponden a este nivel. El sector pone de manifiesto que el cliente y los recursos humanos son dos aspectos clave en el sector hotelero. Prueba de ello es que las dos principales tareas o cuestiones que reciben una mayor importancia, y para la que por tanto consideran que deberían estar formados los egresados, están relacionadas con la medición de la satisfacción del cliente y la planificación y programación de los recursos humanos de la organización.

La definición de una adecuada estrategia de operaciones, objetivos a largo plazo y la cuidad selección y diseño de los servicios a ofrecer son las dos cuestiones que les siguen en importancia y además las más relevantes cuestiones de carácter estratégico. La medición del rendimiento y la productividad, así como la aplicación del Yield Management, dentro de la gestión de la demanda y capacidad a medio y corto plazo, también se alzan como cuestiones que ocupan buena parte de la gestión de estas empresas. En contra de lo que quizás sería de esperar, los temas relacionados con el diseño, gestión y control de la calidad (tanto desde el punto de vista estratégico como táctico/operativo) y el relacionado con la gestión medioambiental, parecen tener para los expertos hoteleros una importancia moderada. Se trata de aspectos que se sitúan hacia el medio de la tabla.

Como ya hemos comentado, los autores se muestran convencidos de que la formación universitaria debe intentar satisfacer las necesidades del sector al que se refieren, de forma que la incorporación de los egresados al mercado laboral se agilice y sea más eficiente. Es por ello que sugerimos el uso de estos resultados en el diseño de planes de estudio y en la configuración de los contenidos de las asignaturas de grado y postgrado en materia turística. De esta forma esperamos, modestamente, acercar la formación e investigación en Dirección de Operaciones y la praxis hotelera.

\section{BIBLIOGRAFÍA}

Álvarez Gil, M.J., De Burgos Jiménez, J, Céspedes Lorente, J.J. (2001): "An analysis of environment management, organizational context and performance of Spanish hotels". Omega, no 29, pp.457-471.

Breiter, D. y Hoart, H. (2000): "Competencies un foodservice information technology expected by the foodservice industry of graduates of hospitality bachelor's degree programs in the United States". Journal of Hospitality \& Tourism Education. Vol. 12. № 2: p11.

Briedenhann, J. (2007): “The Role of the Public Sector in Rural Tourism: Respondents' Views". Current Issues in Tourism, 2007, vol. 10, no 6, p584-607

Ceballos Hernández y Ruiz Jiménez (2009): Dirección de Operaciones en Empresas Turísticas: Enseñanza, Investigación y Necesidades Reales del Sector. Tesis Doctoral. Universidad de Sevilla.

Ceballos Hernández,C.; Giráldez Puig, M.P.; Gonzalez Zamora, M.M. y Ríos Martín, M.A. (2008): "The Influence of Icts on Profitability, Productivity and Quality of Services in Andalusian Hotel Industry", en Innovation, Enterpreneurialship, Knowledge and Enterprise in Competitiveness. Actas de las XVIII Jornadas LusoEspanholas de Gestao Científica, num. 4, pp. 69-69. Oporto, Portugal.

Chan, W.; Wong, K.; Lo, J. (2008): "Environmental quality index for the Hong Kong hotel sector". Tourism Economics, vol. 14, no 4, pp. 857-870.

Chernish, W.N. y Spivack, S.E. (1998): "Assessing the need for mutual understanding: health education for tourism educators and tourism education for health educators". Asia Pacific Journal of Tourism Research. Vol. 3. № 1: pp. 45-54.

De Burgos Jiménez, J, Céspedes Lorente, J.J. y Álvarez Gil, M.J. (2003): "Dirección de operaciones de servicios: los hoteles españoles". Revista Europea de Dirección y Economía de la Empresa, vol.12, no1, pp.83-98.

Fayos-Sola, E. (Ed.) (1997): Introducción a TEDQUAL: una metodología para la calidad en educación y formación turísticas. Organización Mundial del Turismo. Madrid.

Fernández García, M.M.; Campos Soria, J.A.; Maté Jiménez, C. (2001): "La medición de la satisfacción del cliente de hotel. Estado del arte y nuevas perspectivas sobre su medición”. Estudios Turísticos, nº147, pp. 23-56

Gallego Pereira, M.D.; Luna Huertas, P. y Bueno Ávila, S. (2008): "Designing a forecasting analysis to understand the diffusion of open source software in the year 2010". Technological Forecasting and Social Change, vol. 75, nำ5, pp. 672-686.

Garrod, B. y Fyall, A. (2005): "Revisiting Delphi: the Delphi technique in tourism research", en B. Ritchie, P. Burns, y C. Palmer (Eds) Tourism research methods: integrating theory with practice, pp. 85-98. Wallingford: $\mathrm{CABI}$ Publishing.

Gestión de Hoteles (2008): “La formación: Objetivo prioritario para las cadenas hoteleras”. Gestión de hoteles, no 77 , pp. $40-41$. 
Green, H.; Hunter, C. y Moore, B. (1990): "Assessing the environmental impact of tourism development: using the Delphi technique". Tourism Management, vol. 11, №2, pp. 111-120.

Hostetur (2008): "Ranking Hoteltur de cadenas hoteleras españolas 2008". Hosteltur. Septiembre 2008, p. 29.

Hu, B. y Cai, L. (2004): "Hotel Labor Productivity Assessment". Journal of Travel \& Tourism Marketing, vol. 16, no 2-3, pp. $27-38$.

Huete, R. (2007): "Tourism Studies in Spain: The role of Sociology in degree programmes". Journal of Teaching in Travel \& Tourism, vol 7, nำ 2, pp.73-91.

Jones D.; Mak, B. y Sim, J. (2007): "A New Look at the Antecedents and Consequences of Relationship Quality in the Hotel Service Environment". Services Marketing Quarterly, vol. 28, no 3, pp. 15-31.

Kasim, A. (2006): "The Need for Business Environmental and Social Responsibility in the Tourism Industry". International Journal of Hospitality and Tourism Administration, vol. 7, oㅡ 1, pp. 1-22.

Kasim, A. (2007): "Towards a Wider Adoption of Environmental Responsibility in the Hotel Sector". International Journal of Hospitality and Tourism Administration, vol. 8, nำ2, pp. 25-49.

Kerstetter, D. y Cho, M. (2004): "Prior Knowledge, Credibility and Information Search". Annals of Tourism Research, vol. 31, no 4, pp. 961-985.

Kim, KK; O'Bryan, CA; Crandall, PG; Ricke, SC y Neal, JA (2013): "Identifying baseline food safety training practices for retail delis using the Delphi expert consensus method". Food Control, vol. 32, no 1, pp. 5562.

Kunina-Habenicht, O; Lohse-Bossenz, H.; Kunter, M.; et al. (2012): "What educational science content is important for teacher training? - Results of a Delphi study". Zeitschrift Fur Erziehungswissenschaft, vol 15, no 4, pp. 649-682.

Küster, I. y Antón, J.J. (2004): "La formación del factor humano: elemento determinante de la calidad del servicio”. Gestión en H, no 16, marzo-abril, pp. 50-54.

Lillo Bañuls, A.; Ramón Rodríguez, A.B. y Sevilla Jiménez, M. (2007): "El capital Humano como factor estratégico para la competitividad del sector turístico". Cuadernos de Turismo, no 19, pp. 47-69.

Linstone, H. A. y Turoff, M. (1975): “The delphi method: techniques and applications”. Addison-Wesley.

Management Studies, University of Surrey.

Masberg, B. A.; Chase, D.M. y Madlem, M.S. (2003): "A Delphi Study of Tourism Training and Education Needs in Washington State". Journal of Human Resources in Hospitality y Tourism. Vol. 2. № 2.

May, A. K., Cuschieri, J., Johnson, J. L., Duane, T. M., Cherry-Bukowiec, J. R., y Rosengart, M. R. (2013): "Determining a Core Curriculum in Surgical Infections for Fellowship Training in Acute Care Surgery Using the Delphi Technique". Surgical infections, vol. 14, no 6, pp. 547-553.

O'Connor, P. y Frew, A.J. (2004): "An evaluation methodology for hotel electronic channels of distribution". Hospitality Management, nำ 23, pp. 179-199

Onyango, F. E.; Monica, A.; Edwin, O. y Roselyne, O. (2009): "The Role of Human Resource Systems and Competitive Strategies in Hospitality Organizational Performance in Kenya". International Journal of Hospitality and Tourism Administration, vol. 10, no 2, p. 174-194.

Rohan, D.; Ahern, S. y Walsh, K. (2009): "Defining an anaesthetic curriculum for medical undergraduates. A Delphi study". Medical Teacher, vol. 31, no 1, pp. E1-E5.

Sigala, M.; Jones, P.; Lockwood, A. y Airey, D. (2005): Productivity in hotels: a stepwise data envelopment analysis of hotels' rooms division processes. Service Industries Journal, vol. 25, no 1, pp. 61-81.

Spenceley, A. (2008): "Requirements for Sustainable Nature-based Tourism in Transfrontier Conservation Areas: a Southern African Delphi Consultation”. Tourism Geographies, Aug2008, vol. 10, nํㅜ, pp. 285-311.

Sulzer-Azaroff, B.; Fleming, R.; Tupa, M.; Bass, R. y Hamad, Ch. (2008): "Choosing Objectives for a Distance Learning Behavioral Intervention in Autism Curriculum". Focus on Autism and Other Developmental Disabilities, vol. 23, no 1, p. 29-36.

Talón Ballesteros, P.; González Serrano, L. y Figueroa Domecq, C. (2007): "La nueva realidad del sector hotelero español”, en Empresa global y mercados locales. Actas del XXI Congreso Anual AEDEM, vol. 2, pag. 20. Universidad Rey Juan Carlos, Madrid.

Witt, C.A. y Clark, B.R. (1990): "Tourism: the use of production management techniques". The Services Industries Journal, vol. 10, nํ2, pp. 306-319.

Witt, C.A. y Witt, S.F. (1989): "Why productivity in the hotel sector is low?". International Journal of Contemporary Hospitality Management, vol. 1, núm. 2, pp. 28-34. 
Wright, C.M. y Mechling, G. (2002): "The importance of operations management problems in service organizations". Omega, n 30, pp. 77-87.

Zehrer, A. y Mössenlechner, C. (2008): "Industry Relations and Curricula Design in Austrian Tourism Master Programs: A Comparative Analysis”. Journal of Teaching in Travel \& Tourism, vol. 8, no. 1, pp. 73-95.

Zehrer, A., Siller, H., \& Altmann, A. (2006): "A module system in tourism and leisure education-theoretical and practical perspectives", en C. Hu (Ed.), Imagining the future of travel and tourism education. Proceedings of the ISTEE Conference 2006 in Las Vegas, USA, pp. 276-285. St Clair Shores, MI: ISTTE.

Zhou, Z.; Huang, Y; Hsu, M.K. (2008): "Using Data Envelopment Analysis to Evaluate Efficiency: An Exploratory Study of the Chinese Hotel Industry". Journal of Quality Assurance in Hospitality \& Tourism, vol. 9, nº 3, pp. 240-256.

Zolingen, S. y Klaassen, C. (2003): "Selection processes in a Delphi study about key qualifications in Senior Secondary Vocational Education”. Technological Forecasting and Social Change, vol. 70, no 4, pp. 317340. 